\title{
ОПТИМАЛЬНЫЕ СТРАТЕГИИ ЛЕЧЕНИЯ РАКОВЫХ ЗАБОЛЕВАНИЙ В МАТЕМАТИЧЕСКОЙ МОДЕЛИ КОНКУРЕНЦИИ ЛОТКИ - ВОЛЬТЕРРЫ ${ }^{1}$
}

\author{
Н. Л. Григоренко, Е. Н. Хайлов, Э.В.Григорьева, А. Д. Клименкова
}

\begin{abstract}
Для описания взаимодействия концентраций здоровых и раковых клеток при заболеваниях, связанных с раком крови, используется модель конкуренции Лотки - Вольтерры. В эту модель добавляется дифференциальное уравнение, описывающее изменение концентрации химиотерапевтического препарата. Это уравнение содержит скалярное ограниченное управление, которое задает интенсивность поступления такого препарата в организм. Для рассматриваемой управляемой системы ставится задача минимизации взвешенной разности концентраций раковых и здоровых клеток в конечный момент времени заданного периода лечения. С помощью принципа максимума Понтрягина аналитически устанавливаются свойства оптимального управления. Выделяются ситуации, когда такое управление является релейной функцией, а также ситуации, когда наряду с релейными участками оно может также содержать и участок с особым режимом. Полученные результаты подтверждаются соответствующими численными расчетами.

Ключевые слова: модель конкуренции Лотки - Вольтерры, нелинейная управляемая система, принцип максимума Понтрягина, функция переключений, релейное управление, особый режим.

N. L. Grigorenko, E. N. Khailov, E. V. Grigorieva, A. D. Klimenkova. Optimal strategies in the treatment of cancers in the Lotka-Volterra mathematical model of competition.

The Lotka-Volterra competition model is applied to describe the interaction between the concentrations of healthy and cancer cell in diseases associated with blood cancer. The model is supplemented with a differential equation characterizing the change in the concentration of a chemotherapeutic drug. The equation contains a scalar bounded control that specifies the intensity of drug intake. We consider the problem of minimizing the weighted difference between the concentrations of cancer and healthy cells at the end time of the treatment period. The properties of an optimal control are established analytically with the use of the Pontryagin maximum principle. We describe situations in which the optimal control is a relay function and situations in which the control may contain a segment with a singular arc in addition to relay segments. The results obtained are confirmed by corresponding numerical calculations.
\end{abstract}

Keywords: Lotka-Volterra competition model, nonlinear control system, Pontryagin maximum principle, switching function, bang-bang control, singular arc.

MSC: 49K15, 93A30

DOI: $10.21538 / 0134-4889-2020-26-1-71-88$

\section{Введение}

По данным Всемирной Организации Здравоохранения (ВО3), каждый год от рака умирает около 7.6 миллионов человек, что составляет $13 \%$ в структуре общей смертности в мире. На лечение раковых заболеваний тратятся миллиарды долларов. На сегодняшний день известно, что рак - это не одна болезнь, а, по крайней мере, двести, причем каждая из них имеет свои симптомы, методы диагностики и лечения. ВОЗ считает, что число случаев заболеваний раком возрастет в ближайшие двадцать лет на $70 \%$. Одним из характерных признаков рака является быстрое образование аномальных клеток, прорастающих за пределы своих обычных границ и способных проникать в близлежащие части тела и распространяться в другие органы.

Среди всевозможных раковых заболеваний особое место занимает рак крови, объединяющий в себе раковые заболевания кроветворной (лейкемия), лимфатической (лимфома) систем и плазмы крови (миелома). На сегодняшний день эти заболевания считаются неизлечимыми.

\footnotetext{
${ }^{1}$ Работа первых двух авторов выполнена при финансовой поддержке РФФИ и ДНТ в рамках научного проекта 18-51-45003 ИНД_а.
} 
Однако с помощью современных методов лечения зачастую удается добиться полной ремиссии, избавить пациента от симптомов и улучшить качество его жизни. Такие заболевания удается доводить до состояния, близкого к хронической форме; их можно лечить при каждом рецидиве. При этом значительный вклад здесь вносит математическое моделирование, позволяющее описывать поведение клеток и органов до заболевания, при его развитии и лечении, обходясь без сложнейших и дорогостоящих наблюдений. При отыскании эффективных в том или ином смысле стратегий лечения широко применяется математическая теория оптимального управления.

Не приводя здесь полный обзор работ, связанных как с математическим моделированием лейкемии, лимфомы и миеломы, так и с поиском эффективных стратегий их лечения на основе теории оптимального управления, мы бы хотели выделить работы [1-6], которые по ряду причин важны для нас. Во-первых, в этих работах такие обзоры представлены. Во-вторых, взаимодействие между здоровыми и раковыми клетками в них описывается с помощью обыкновенных дифференциальных уравнений, использующих логистический закон и закон Гомперца. В-третьих, в них осуществляется поиск как оптимальных, так и альтернативных стратегий лечения указанных заболеваний.

Для описания взаимодействия концентраций здоровых и раковых клеток при лейкемии, лимфоме и миеломе также может быть использована и математическая модель конкуренции Лотки-Вольтерры [7-9], которая тоже записывается с помощью обыкновенных дифференциальных уравнений. Работа [10] демонстрирует применение теории оптимального управления в такой модели. Настоящая работа является ее продолжением. В отличие от [10], в эту модель добавлено дифференциальное уравнение, описывающее изменение концентрации химиотерапевтического препарата. Это уравнение содержит скалярное ограниченное управление, которое задает интенсивность поступления такого препарата в организм. Эффективность применяемого лечения описывается с помощью монотонной функции терапии. Для рассматриваемой управляемой системы ставится задача минимизации терминального функционала, представляющего собой взвешенную разность концентраций раковых и здоровых клеток в конечный момент времени заданного периода лечения. С использованием принципа максимума Понтрягина аналитически устанавливаются свойства оптимального управления. Выделены ситуации, когда такое управление является релейной функцией на всем отрезке времени, а также ситуации, когда наряду с релейными участками оно может содержать и особый участок с особым режимом. Полученные свойства оптимального управления подтверждаются соответствующими численными расчетами, выполненными в среде ВОСОР-2.0.5.

\section{1. Постановка задачи минимизации}

Будем использовать для описания взаимодействия между популяциями здоровых и раковых клеток математическую модель конкуренции Лотки - Вольтерры [11, с. 121; 12, с. 43]

$$
\left\{\begin{array}{l}
N_{1}^{\prime}(\tau)=r_{1}\left(1-K_{1}^{-1} N_{1}(\tau)-a_{12} K_{2}^{-1} N_{2}(\tau)\right) N_{1}(\tau) \\
N_{2}^{\prime}(\tau)=r_{2}\left(1-K_{2}^{-1} N_{2}(\tau)-a_{21} K_{1}^{-1} N_{1}(\tau)\right) N_{2}(\tau)
\end{array}\right.
$$

Как уже отмечалось ранее, эта модель удобна для математического описания таких раковых заболеваний, как лейкемия, лимфома, миелома (рак крови). Здесь $N_{1}(\tau)$ и $N_{2}(\tau)$ - количество здоровых и раковых клеток в момент времени $\tau \geq 0$ соответственно. Величины $r_{1}, r_{2}, K_{1}$, $K_{2}, a_{12}, a_{21}$ являются положительными параметрами системы (1.1): $r_{1}$ и $r_{2}$ суть внутренние скорости роста, а $K_{1}$ и $K_{2}$ - максимальные размеры соответствующих популяций. Наконец, $a_{12}$ - скорость инактивации здоровых клеток раковыми и $a_{21}-$ скорость инактивации раковых клеток здоровыми. Анализ устойчивости положений равновесия системы (1.1) подробно изложен в $[11$, с. $121 ; 12$, с. 43$]$. 
Уменьшим количество параметров рассматриваемой модели с шести до трех с помощью следующего масштабирования и переобозначения фазовых переменных и параметров системы:

$$
x=K_{1}^{-1} N_{1}, \quad y=K_{2}^{-1} N_{2}, \quad t=r_{2} \tau, \quad r=r_{1} r_{2}^{-1} .
$$

Тогда система (1.1) примет вид

$$
\left\{\begin{array}{l}
x^{\prime}(t)=r\left(1-x(t)-a_{12} y(t)\right) x(t), \\
y^{\prime}(t)=\left(1-y(t)-a_{21} x(t)\right) y(t) .
\end{array}\right.
$$

В этой системе мы считаем $x(t)$ и $y(t)$ концентрациями здоровых и раковых клеток в момент времени $t \geq 0$ соответственно. Величины $r, a_{12}, a_{21}$ являются положительными параметрами системы (1.2): $r$ есть внутренняя скорость роста здоровых клеток. Смысл $a_{12}$ и $a_{21}$ не меняется. Именно, $a_{12}$ - скорость инактивации здоровых клеток раковыми и $a_{21}-$ скорость инактивации раковых клеток здоровыми. Выбор значений параметров $r, a_{12}, a_{21}$ представляет самостоятельную задачу и определяется конкретным видом ракового заболевания. Мы также считаем, что в дальнейших рассуждениях справедливо неравенство

$$
a_{12} \cdot a_{21} \neq 1 \text {. }
$$

Добавим в уравнения системы (1.2) слагаемые, отражающие влияние химиотерапевтического воздействия на здоровые и раковые клетки при лечении, убивающем раковые клетки. В результате приходим к следующей системе:

$$
\left\{\begin{array}{l}
x^{\prime}(t)=r\left(1-x(t)-a_{12} y(t)\right) x(t)-m_{1} g(z(t)) x(t), \\
y^{\prime}(t)=\left(1-y(t)-a_{21} x(t)\right) y(t)-m_{2} g(z(t)) y(t) .
\end{array}\right.
$$

Здесь $z(t)$ - концентрация химиотерапевтического препарата в момент времени $t \geq 0$, a $g(z)-$ функция терапии, которая непосредственно задает влияние химиотерапевтического воздействия на здоровые и раковые клетки. Положительные величины $m_{1}$ и $m_{2}$ определяют уровни смертности популяций соответственно здоровых и раковых клеток во время лечения. В дальнейших рассуждениях мы также считаем выполненным неравенство

$$
m_{2}>m_{1}
$$

отражающее более сильное воздействие применяемого лечения на популяцию раковых клеток, чем на популяцию здоровых клеток.

Будем предполагать, что $g(z)$ - достаточно гладкая, монотонно возрастающая функция: $g(z)>0$ при $z>0$ и $g(0)=0 ; g^{\prime}(z)>0$ при $z \geq 0$. Примерами таких функций являются $g(z)=z$ и $g(z)=z(\mu+z)^{-1}$, где $\mu-$ заданная положительная константа. Более подробно такие функции терапии обсуждаются в $[2 ; 3 ; 5]$.

Теперь добавим к системе (1.4) уравнение, описывающее изменение концентрации химиотерапевтического препарата с течением времени:

$$
z^{\prime}(t)=-\gamma z(t)+u(t)
$$

где $u(t)$ - управление, характеризующее интенсивность поступления химиотерапевтического средства в момент времени $t \geq 0$, и $\gamma$ - коэффициент диссипации. Пусть множество допустимых управлений $\Omega(T)$ образуют всевозможные измеримые по Лебегу функции $u(t)$, которые при почти всех $t \geq 0$ удовлетворяют ограничениям

$$
0 \leq u(t) \leq M,
$$

где $M$ - заданная положительная константа. 
Начальные условия для уравнений системы (1.4) и (1.6) имеют вид

$$
x(0)=x_{0}, \quad y(0)=y_{0}, \quad z(0)=0,
$$

где величины $x_{0}$ и $y_{0}$ удовлетворяют включениям

$$
x_{0}, y_{0} \in(0,1) .
$$

Пусть задан отрезок времени $[0, T]$, определяющий длительность периода лечения. Зададим произвольное управление $u(\cdot) \in \Omega(T)$. Тогда, благодаря (1.8) решение $z(t)$ уравнения (1.6) определено на всем отрезке $[0, T]$ и выражается формулой

$$
z(t)=e^{-\gamma t} \int_{0}^{t} e^{\gamma s} u(s) d s, \quad t \in[0, T] .
$$

Из ограничений (1.7) и формулы (1.10) вытекают неравенства

$$
0 \leq z(t) \leq \gamma^{-1} M\left(1-e^{-\gamma t}\right) \leq \gamma^{-1} M, \quad t \in[0, T] .
$$

Теперь подставим функцию $z(t)$ в уравнения системы (1.4). Привлекая соответствующие начальные условия из (1.8), имеем решение $(x(t), y(t))$ этой системы, определенное на некотором отрезке $\left[0, T_{0}\right]$. Перепишем уравнения системы (1.4) как линейные однородные дифференциальные уравнения вида

$$
\begin{aligned}
& x^{\prime}(t)=\left[r\left(1-x(t)-a_{12} y(t)\right)-m_{1} g(z(t))\right] x(t)=P(t) x(t), \\
& y^{\prime}(t)=\left[\left(1-y(t)-a_{21} x(t)\right)-m_{2} g(z(t))\right] y(t)=Q(t) y(t) .
\end{aligned}
$$

Учитывая соответствующие начальные условия из (1.8), мы видим, что справедливы равенства

$$
x(t)=x_{0} e^{\int^{t} P(s) d s}, \quad y(t)=y_{0} e^{\int^{t} Q(s) d s}, \quad t \in\left[0, T_{0}\right],
$$

из которых немедленно следует положительность функций $x(t)$ и $y(t)$ всюду на отрезке $\left[0, T_{0}\right]$.

Благодаря этому свойству, а также вытекающей из (1.11) неотрицательности функции $z(t)$ находим дифференциальные неравенства

$$
x^{\prime}(t) \leq r(1-x(t)) x(t), \quad y^{\prime}(t) \leq(1-y(t)) y(t),
$$

являющиеся следствием соответствующих уравнений системы (1.4).

Далее мы будем рассматривать только первое неравенство. Рассуждения для второго неравенства проводятся аналогично. Перепишем первое неравенство в (1.12) в виде

$$
\frac{x^{\prime}(t)}{x^{2}(t)} \leq \frac{r}{x(t)}-r, \text { или }\left(\frac{e^{r t}}{x(t)}\right)^{\prime} \geq r e^{r t} .
$$

Интегрируя это неравенство на отрезке $[0, t]$ с соответствующим начальным условием из (1.8), получаем неравенство

$$
x(t) \leq \frac{x_{0}}{x_{0}+\left(1-x_{0}\right) e^{-r t}} .
$$

При $t=0$ его правая часть равна $x_{0}$. Согласно соответствующему включению из (1.9) правая часть неравенства является монотонно возрастающей до значения 1 при $t \rightarrow+\infty$ функцией. Поэтому $x(t) \in(0,1)$ при всех $t \in\left[0, T_{0}\right]$. Следовательно, имеет место и включение $y(t) \in(0,1)$ для всех $t \in\left[0, T_{0}\right]$. С учетом [13, с. 26] эти включения гарантируют продолжимость решения $(x(t), y(t))$ системы (1.4) на отрезок времени $[0, T]$ в случае $T_{0}<T$. Если $T_{0} \geq T$, то такие включения уже имеют место на отрезке $[0, T]$.

Таким образом, нами установлена справедливость следующей леммы. 
Лемма 1. Для любого управления $u(\cdot) \in \Omega(T)$ соответствующее решение $(x(t), y(t), z(t))$ уравнений системы (1.4) и (1.6) с начальными условиями (1.8) определено на всем отрезке $[0, T]$ и удовлетворяет включению

$$
(x(t), y(t), z(t)) \in \Lambda, \quad t \in[0, T],
$$

где

$$
\Lambda=\left\{(x, y, z): 0<x<1,0<y<1,0 \leq z \leq \gamma^{-1} M\right\} .
$$

Для уравнений системы (1.4) и (1.6) с начальными условиями (1.8) на множестве допустимых управлений $\Omega(T)$ рассмотрим задачу минимизации функционала

$$
J(u(\cdot))=y(T)-\alpha x(T),
$$

который представляет собой взвешенную разность концентраций раковых и здоровых клеток в конечный момент времени $T$ периода лечения $[0, T]$. Здесь $\alpha$ есть положительный весовой коэффициент. При этом если $\alpha>1$, то оказывается предпочтительнее максимизировать концентрацию здоровых клеток $x(T)$; если же $\alpha<1$, то важной становится минимизация концентрации раковых клеток $y(T)$.

В силу леммы 1 и теоремы 4 [14, гл. 4] в рассматриваемой задаче минимизации существует оптимальное решение, состоящее:

- из оптимального управления $u_{*}(t)$;

- из соответствующего оптимального решения $\left(x_{*}(t), y_{*}(t), z_{*}(t)\right)$ уравнений системы $(1.4)$ и (1.6) с начальными условиями (1.8).

\section{2. Принцип максимума Понтрягина}

Для анализа оптимального решения, состоящего из управления $u_{*}(t)$ и отвечающего ему решения $\left(x_{*}(t), y_{*}(t), z_{*}(t)\right)$, применим принцип максимума Понтрягина [15, гл. 6, теорема 1$]$.

Сначала выпишем функцию Гамильтона - Понтрягина

$H\left(x, y, z, u, \psi_{1}, \psi_{2}, \psi_{3}\right)=\left(r\left(1-x-a_{12} y\right)-m_{1} g(z)\right) x \psi_{1}+\left(\left(1-y-a_{21} x\right)-m_{2} g(z)\right) y \psi_{2}+(-\gamma z+u) \psi_{3}$,

где $\psi_{1}, \psi_{2}, \psi_{3}$ - сопряженные переменные.

Теперь вычислим требуемые частные производные функции $H\left(x, y, z, u, \psi_{1}, \psi_{2}, \psi_{3}\right)$

$$
\begin{aligned}
& H_{x}^{\prime}\left(x, y, z, u, \psi_{1}, \psi_{2}, \psi_{3}\right)=\left(r\left(1-x-a_{12} y\right)-m_{1} g(z)\right) \psi_{1}-r x \psi_{1}-a_{21} y \psi_{2}, \\
& H_{y}^{\prime}\left(x, y, z, u, \psi_{1}, \psi_{2}, \psi_{3}\right)=\left(\left(1-y-a_{21} x\right)-m_{2} g(z)\right) \psi_{2}-r a_{12} x \psi_{1}-y \psi_{2}, \\
& H_{z}^{\prime}\left(x, y, z, u, \psi_{1}, \psi_{2}, \psi_{3}\right)=-g^{\prime}(z)\left(m_{1} x \psi_{1}+m_{2} y \psi_{2}\right)-\gamma \psi_{3}, \\
& H_{u}^{\prime}\left(x, y, z, u, \psi_{1}, \psi_{2}, \psi_{3}\right)=\psi_{3} .
\end{aligned}
$$

Тогда в соответствии с принципом максимума Понтрягина существует вектор-функция $\psi(t)=\left(\psi_{1}(t), \psi_{2}(t), \psi_{3}(t)\right)$ такая, что:

1) функция $\psi(t)$ является решением сопряженной системы

$$
\left\{\begin{aligned}
\psi_{1}^{\prime}(t)= & -H_{x}^{\prime}\left(x_{*}(t), y_{*}(t), z_{*}(t), u_{*}(t), \psi_{1}(t), \psi_{2}(t), \psi_{3}(t)\right) \\
= & -\left(r\left(1-x_{*}(t)-a_{12} y_{*}(t)\right)-m_{1} g\left(z_{*}(t)\right)\right) \psi_{1}(t) \\
& +r x_{*}(t) \psi_{1}(t)+a_{21} y_{*}(t) \psi_{2}(t) \\
\psi_{2}^{\prime}(t)= & -H_{y}^{\prime}\left(x_{*}(t), y_{*}(t), z_{*}(t), u_{*}(t), \psi_{1}(t), \psi_{2}(t), \psi_{3}(t)\right) \\
= & -\left(\left(1-y_{*}(t)-a_{21} x_{*}(t)\right)-m_{2} g\left(z_{*}(t)\right)\right) \psi_{2}(t) \\
& +r a_{12} x_{*}(t) \psi_{1}(t)+y_{*}(t) \psi_{2}(t) \\
\psi_{3}^{\prime}(t)= & -H_{z}^{\prime}\left(x_{*}(t), y_{*}(t), z_{*}(t), u_{*}(t), \psi_{1}(t), \psi_{2}(t), \psi_{3}(t)\right) \\
= & g^{\prime}\left(z_{*}(t)\right)\left(m_{1} x_{*}(t) \psi_{1}(t)+m_{2} y_{*}(t) \psi_{2}(t)\right)+\gamma \psi_{3}(t), \\
\psi_{1}(T)= & \alpha, \psi_{2}(T)=-1, \psi_{3}(T)=0
\end{aligned}\right.
$$


2) управление $u_{*}(t)$ максимизирует функцию Гамильтона - Понтрягина

$$
H\left(x_{*}(t), y_{*}(t), z_{*}(t), u, \psi_{1}(t), \psi_{2}(t), \psi_{3}(t)\right)
$$

по переменной $u \in[0, M]$ при почти всех $t \in[0, T]$, а потому оно удовлетворяет следующему соотношению:

$$
u_{*}(t)= \begin{cases}M, & \text { если } L_{u}(t)>0, \\ \text { любое } u \in[0, M], & \text { если } L_{u}(t)=0, \\ 0, & \text { если } L_{u}(t)<0\end{cases}
$$

где $L_{u}(t)=\psi_{3}(t)$ есть функция переключений, которая описывает поведение управления $u_{*}(t)$ согласно формуле $(2.2)$.

Теперь преобразуем дифференциальные уравнения системы (2.1) вместе с отвечающими им начальными условиями, введя в рассмотрение новые сопряженные переменные

$$
\phi_{1}(t)=-x_{*}(t) \psi_{1}(t), \quad \phi_{2}(t)=-y_{*}(t) \psi_{2}(t), \quad \phi_{3}(t)=\psi_{3}(t) .
$$

Выполняя необходимые вычисления, имеем новую сопряженную систему

$$
\left\{\begin{array}{l}
\phi_{1}^{\prime}(t)=r x_{*}(t) \phi_{1}(t)+a_{21} x_{*}(t) \phi_{2}(t), \\
\phi_{2}^{\prime}(t)=r a_{12} y_{*}(t) \phi_{1}(t)+y_{*}(t) \phi_{2}(t), \\
\phi_{3}^{\prime}(t)=\gamma \phi_{3}(t)-g^{\prime}\left(z_{*}(t)\right)\left(m_{1} \phi_{1}(t)+m_{2} \phi_{2}(t)\right), \\
\phi_{1}(T)=-\alpha x_{*}(T), \phi_{2}(T)=y_{*}(T), \phi_{3}(T)=0 .
\end{array}\right.
$$

При этом функция переключений $L_{u}(t)$ будет определяться через новую переменную $\phi_{3}(t)$ формулой $L_{u}(t)=\phi_{3}(t)$.

Учитывая этот факт, перепишем новую сопряженную систему (2.3) в виде

$$
\left\{\begin{array}{l}
\phi_{1}^{\prime}(t)=r x_{*}(t) \phi_{1}(t)+a_{21} x_{*}(t) \phi_{2}(t), \\
\phi_{2}^{\prime}(t)=r a_{12} y_{*}(t) \phi_{1}(t)+y_{*}(t) \phi_{2}(t), \\
L_{u}^{\prime}(t)=\gamma L_{u}(t)-g^{\prime}\left(z_{*}(t)\right)\left(m_{1} \phi_{1}(t)+m_{2} \phi_{2}(t)\right), \\
\phi_{1}(T)=-\alpha x_{*}(T), \phi_{2}(T)=y_{*}(T), L_{u}(T)=0 .
\end{array}\right.
$$

Установим важное свойство новых сопряженных переменных $\phi_{1}(t), \phi_{2}(t)$ с помощью следующей леммы.

Лемма 2. Сопряжсеные переменные $\phi_{1}(t)$ и $\phi_{2}(t)$ знакоопределены на отрезке $[0, T]$, m. е. справедливы неравенства

$$
\phi_{1}(t)<0, \quad \phi_{2}(t)>0, \quad t \in[0, T] .
$$

Д о к а з а т е л ь с т в о. Из леммы 1 и соответствующих начальных условий из (2.4) вытекают неравенства

$$
\phi_{1}(T)<0, \quad \phi_{2}(T)>0 .
$$

Далее, не существует такого значения $t_{0} \in[0, T)$, при котором одновременно выполнены равенства $\phi_{1}\left(t_{0}\right)=0, \phi_{2}\left(t_{0}\right)=0$. В противном случае, поскольку первые два дифференциальных уравнения системы (2.4) образуют линейную однородную систему, то $\phi_{1}(t)=0$ и $\phi_{2}(t)=0$ при всех $t \in[0, T]$, что противоречит неравенствам (2.6). Также из анализа этих неравенств мы заключаем, что возможны следующие два случая.

С л у ч а й 1 . Пусть определено такое значение $t_{1} \in[0, T)$, что

$$
\phi_{1}\left(t_{1}\right)=0
$$


и справедливы соотношения

$$
\begin{array}{lll}
\phi_{1}(t)<0 & \text { при } & t \in\left(t_{1}, T\right], \\
\phi_{2}(t)>0 & \text { при } & t \in\left[t_{1}-\epsilon_{1}, T\right]
\end{array}
$$

для некоторого малого $\epsilon_{1}>0$. Подставляя (2.7) в первое уравнение системы $(2.4)$ и учитывая при этом лемму 1 и неравенство (2.9), получаем $\phi_{1}^{\prime}\left(t_{1}\right)=a_{21} x_{*}\left(t_{1}\right) \phi_{2}\left(t_{1}\right)>0$, которое вместе с равенством (2.7) означает, что функция $\phi_{1}(t)$ при переходе через точку $t_{1}$ меняет свой знак с отрицательного на положительный, что противоречит (2.8). Значит, такой случай невозможен.

С л у ч а й 2. Пусть определено такое значение $t_{2} \in[0, T)$, что

$$
\phi_{2}\left(t_{2}\right)=0
$$

и выполнены соотношения

$$
\begin{array}{lll}
\phi_{1}(t)<0 & \text { при } & t \in\left[t_{2}-\epsilon_{2}, T\right], \\
\phi_{2}(t)>0 & \text { при } & t \in\left(t_{2}, T\right],
\end{array}
$$

где $\epsilon_{2}$ - малое положительное число. Подставляя (2.10) во второе уравнение системы (2.4) и учитывая при этом лемму 1 и неравенство $(2.11)$, находим $\phi_{2}^{\prime}\left(t_{2}\right)=r a_{12} y_{*}\left(t_{2}\right) \phi_{1}\left(t_{2}\right)<0$, которое вместе с равенством (2.10) означает, что функция $\phi_{2}(t)$ при переходе через точку $t_{2}$ меняет свой знак с положительного на отрицательный, что противоречит (2.12). Поэтому и такой случай также невозможен.

Невозможность случаев 1 и 2 позволяет нам сделать вывод, что переменные $\phi_{1}(t)$ и $\phi_{2}(t)$ не обращаются в нуль всюду на отрезке $[0, T]$. Тогда неравенства (2.6) приводят к выполнению требуемых соотношений (2.5). Утверждение доказано.

Наконец, рассмотрим вместе формулу (2.2) и уравнения системы (2.4). Их анализ показывает, каким может быть поведение функции переключений $L_{u}(t)$, а значит и отвечающего ей оптимального управления $u_{*}(t)$. Поскольку $L_{u}(t)$ является непрерывно-дифференцируемой функцией, то управление $u_{*}(t)$ может иметь релейный вид и переключаться между значениями 0 и $M$. Это будет происходить, если при переходе через значения $t$, в которых функция $L_{u}(t)$ обращается в нуль, имеет место смена знака этой функции. Такие значения $t$ являются переключениями оптимального управления $u_{*}(t)$. Помимо участков релейного типа управление $u_{*}(t)$ может содержать также и особые участки, на которых имеют место особые режимы (singular arcs) [16, гл. 2.8; 17, с. 393; 18]. Такое происходит, когда функция переключений $L_{u}(t)$ обращается тождественно в нуль на некоторых интервалах отрезка $[0, T]$.

Следующий раздел как раз посвящен подробному изучению возможного существования особого режима у оптимального управления $u_{*}(t)$, а также выяснению особенностей релейного управления $u_{*}(t)$.

\section{3. Свойства оптимального управления}

Выделим из системы (2.4) дифференциальное уравнение для функции переключений $L_{u}(t)$ :

$$
L_{u}^{\prime}(t)=\gamma L_{u}(t)-g^{\prime}\left(z_{*}(t)\right)\left(m_{1} \phi_{1}(t)+m_{2} \phi_{2}(t)\right)
$$

с соответствующим начальным условием

$$
L_{u}(T)=0
$$

Продифференцируем это уравнение, используя первые два уравнения системы (2.4). В результате получаем дифференциальное уравнение 


$$
\begin{gathered}
L_{u}^{\prime \prime}(t)=\gamma L_{u}^{\prime}(t)-g^{\prime \prime}\left(z_{*}(t)\right) z_{*}^{\prime}(t)\left(m_{1} \phi_{1}(t)+m_{2} \phi_{2}(t)\right) \\
-g^{\prime}\left(z_{*}(t)\right)\left(r\left(m_{1} x_{*}(t)+a_{12} m_{2} y_{*}(t)\right) \phi_{1}(t)+\left(a_{21} m_{1} x_{*}(t)+m_{2} y_{*}(t)\right) \phi_{2}(t)\right) .
\end{gathered}
$$

Далее сначала найдем из уравнения (3.1) выражение $\left(m_{1} \phi_{1}(t)+m_{2} \phi_{2}(t)\right)$ и подставим во второе слагаемое уравнения (3.3). Затем выделим из (3.1) выражение $g^{\prime}\left(z_{*}(t)\right) \phi_{1}(t)$ и подставим в третье слагаемое этого уравнения. В итоге мы получим линейное неоднородное дифференциальное уравнение второго порядка для функции переключений $L_{u}(t)$ :

$$
L_{u}^{\prime \prime}(t)-(\gamma+b(t)) L_{u}^{\prime}(t)+\gamma b(t) L_{u}(t)=d(t), \quad t \in[0, T]
$$

где

$$
\begin{aligned}
b(t) & =\left(g^{\prime}\left(z_{*}(t)\right)\right)^{-1} g^{\prime \prime}\left(z_{*}(t)\right) z_{*}^{\prime}(t)+r m_{1}^{-1}\left(m_{1} x_{*}(t)+a_{12} m_{2} y_{*}(t)\right), \\
d(t) & =m_{1}^{-1} g^{\prime}\left(z_{*}(t)\right)\left(m_{1}\left(r m_{2}-a_{21} m_{1}\right) x_{*}(t)+m_{2}\left(r a_{12} m_{2}-m_{1}\right) y_{*}(t)\right) \phi_{2}(t) .
\end{aligned}
$$

Рассмотрим отвечающее уравнению (3.4) однородное дифференциальное уравнение

$$
H^{\prime \prime}(t)-(\gamma+b(t)) H^{\prime}(t)+\gamma b(t) H(t)=0, \quad t \in[0, T] .
$$

Легко видеть, что функция $v(t)=e^{\gamma t}$ положительна и является решением этого уравнения. Поэтому на отрезке $[0, T]$ справедлив критерий неосцилляции Валле - Пуссена [19, с. 53], а значит, любое нетривиальное решение уравнения (3.5) имеет на этом отрезке не более одного нуля (говорят, что такое уравнение является неосциллирующим). Тогда для его левой части, а следовательно и для левой части уравнения (3.4), имеет место разложение Пойа Маммана [19, с. 45]:

$$
\rho_{2}(t) \frac{d}{d t}\left(\rho_{1}(t) \frac{d}{d t}\left(\rho_{0}(t) L_{u}(t)\right)\right)=d(t), \quad t \in[0, T]
$$

где $\rho_{i}(t), i=0,1,2$, являются положительными функциями, заданными формулами

$$
\rho_{0}(t)=e^{\gamma(T-t)}, \quad \rho_{1}(t)=e^{\int_{t}^{T} b(s) d s-\gamma(T-t)}, \quad \rho_{2}(t)=e^{-\int_{t}^{T} b(s) d s} .
$$

Теперь пусть для параметров $r, a_{12}, a_{21}, m_{1}, m_{2}$ выполнены либо неравенства

$$
r m_{2}-a_{21} m_{1} \geq 0, \quad r a_{12} m_{2}-m_{1} \geq 0
$$

либо неравенства

$$
r m_{2}-a_{21} m_{1} \leq 0, \quad r a_{12} m_{2}-m_{1} \leq 0 .
$$

Легко видеть, что благодаря (1.3) неравенства в (3.7) и (3.8) одновременно не обращаются в равенства. Поэтому функция $d(t)$ принимает на отрезке $[0, T]$ только положительные значения, если справедливы неравенства (3.7), или только отрицательные значения, если имеют место неравенства (3.8). Тогда, применяя в разложении (3.6) обобщенную теорему Ролля [19, с. 45], мы заключаем, что функция переключений $L_{u}(t)$ имеет на отрезке $[0, T]$ не более двух различных нулей. Значит, благодаря (3.2) эта функция имеет на полуинтервале $[0, T)$ не более одного нуля.

Перепишем дифференциальное уравнение (3.4) в виде

$$
\left(L_{u}^{\prime}(t)-\gamma L_{u}(t)\right)^{\prime}-b(t)\left(L_{u}^{\prime}(t)-\gamma L_{u}(t)\right)=d(t) .
$$

После чего введем новую функцию переключений $G_{u}(t)$ формулой $G_{u}(t)=e^{\gamma(T-t)} L_{u}(t)$. Легко убедиться, что функции $L_{u}(t)$ и $G_{u}(t)$ при одних и тех же значениях $t$ обращаются в нуль, а также для одних и тех же значений $t$ принимают положительные и отрицательные значения. 
Поэтому новая функция переключений $G_{u}(t)$ задает оптимальное управление $u_{*}(t)$ соотношением

$$
u_{*}(t)= \begin{cases}M, & \text { если } G_{u}(t)>0, \\ \text { любое } u \in[0, M], & \text { если } G_{u}(t)=0, \\ 0, & \text { если } G_{u}(t)<0,\end{cases}
$$

которое подобно (2.2).

Тогда перепишем уравнение (3.9) следующим образом:

$$
G_{u}^{\prime \prime}(t)+(\gamma-b(t)) G_{u}^{\prime}(t)=e^{\gamma(T-t)} d(t) .
$$

Пусть $\theta \in[0, T)$ - нуль функции переключений $L_{u}(t)$. Благодаря $(3.2)$ функция $L_{u}(t)$, а значит, и функция $G_{u}(t)$ имеют два различных нуля на отрезке $[0, T]$. Тогда в силу обычной теоремы Ролля между ними лежит нуль $\chi \in[0, T]$ производной $G_{u}^{\prime}(t)$, т. е.

$$
G_{u}^{\prime}(\chi)=0
$$

и в этой точке функция $G_{u}(t)$ достигает либо локального минимума, либо локального максимума. Учитывая (3.12) в уравнении (3.11), находим

$$
G_{u}^{\prime \prime}(\chi)=e^{\gamma(T-\chi)} d(\chi) .
$$

Следовательно, в точке $\chi$ при выполнении неравенств (3.7) функция переключений $G_{u}(t)$ имеет локальный минимум, а при выполнении неравенств (3.8) - локальный максимум.

В силу формулы (3.10) из проделанных рассуждений мы заключаем, что справедлива следующая лемма.

Лемма 3. Имеет место одно из следующих утверждений.

1. При выполнении неравенств (3.7) оптимальное управление $u_{*}(t)$ является либо постоянной функиией, принимающей значение 0 или $M$, либо кусочно-постоянной функиией $c$ одним переключением $\theta_{*} \in(0, T)$ вида

$$
u_{*}(t)=\left\{\begin{array}{cc}
M, & \text { если } 0 \leq t \leq \theta_{*}, \\
0, & \text { если } \theta_{*}<t \leq T .
\end{array}\right.
$$

2. При выполнении неравенств (3.8) оптимальное управление $u_{*}(t)$ является либо постоянной функиией, принимающей значение 0 или $M$, либо кусочно-постоянной функиией $c$ одним переключением $\theta_{*} \in(0, T)$ вида

$$
u_{*}(t)=\left\{\begin{array}{cc}
0, & \text { если } 0 \leq t \leq \theta_{*}, \\
M, & \text { если } \theta_{*}<t \leq T .
\end{array}\right.
$$

Пусть теперь для параметров $r, a_{12}, a_{21}, m_{1}, m_{2}$ справедливы неравенства, отличные от (3.7) и (3.8). Именно мы считаем выполненными либо неравенства

$$
r m_{2}-a_{21} m_{1}>0, \quad r a_{12} m_{2}-m_{1}<0,
$$

либо неравенства

$$
r m_{2}-a_{21} m_{1}<0, \quad r a_{12} m_{2}-m_{1}>0 .
$$

Тогда функция $d(t)$ может обращаться в нуль и, например, иметь конечное число нулей на интервале $(0, T)$. Это приведет к росту числа нулей функции переключений $L_{u}(t)$ и одновременно к росту числа переключений соответствующего оптимального управления $u_{*}(t)$. Заметим, что обобщенная теорема Ролля, примененная опять к разложению (3.6), и равенство (3.2) приведут к выводу о связи числа нулей функции $L_{u}(t)$ на полуинтервале $[0, T)$ с числом нулей 
функции $d(t)$ на интервале $(0, T)$. Именно если функция $d(t)$ имеет $k$ различных нулей на $(0, T)$, то функция переключений $L_{u}(t)$ будет иметь не более $(k+2)$ различных нулей на $[0, T)$.

Другая ситуация для функции $d(t)$ возникает, когда функция переключений $L_{u}(t)$ тождественно обращается в нуль на некотором интервале отрезка $[0, T]$. В такой ситуации оптимальное управление $u_{*}(t)$ может иметь особый режим на этом интервале, который тогда называется особым участком [16, гл. $2.8 ; 17$, с. $393 ; 18]$. Рассмотрим эту ситуацию подробнее.

Пусть функция переключений $L_{u}(t)$ равна тождественно нулю на некотором интервале $\Delta \subset[0, T]$. Тогда первая производная $L_{u}^{\prime}(t)$ функции $L_{u}(t)$ обращается в нуль всюду на этом интервале. Из уравнения (3.1) и монотонности функции $g(z)$ вытекает

$$
m_{1} \phi_{1}(t)+m_{2} \phi_{2}(t)=0, \quad t \in \Delta .
$$

Поэтому и вторая производная $L_{u}^{\prime \prime}(t)$ функции переключений $L_{u}(t)$ также обращается в нуль на интервале $\Delta$. Значит, из уравнения (3.3) и монотонности функции $g(z)$ мы можем вывести равенство

$$
r\left(m_{1} x_{*}(t)+a_{12} m_{2} y_{*}(t)\right) \phi_{1}(t)+\left(a_{21} m_{1} x_{*}(t)+m_{2} y_{*}(t)\right) \phi_{2}(t)=0, \quad t \in \Delta .
$$

Рассмотрим при каждом $t \in \Delta$ соотношения (3.17) и (3.18) как систему линейных алгебраических уравнений, которая в силу леммы 2 имеет нетривиальное решение $\left(\phi_{1}(t), \phi_{2}(t)\right)$. Тогда определитель матрицы такой системы при всех $t \in \Delta$ равен нулю. Это приводит к равенству

$$
m_{1}\left(r m_{2}-a_{21} m_{1}\right) x_{*}(t)+m_{2}\left(r a_{12} m_{2}-m_{1}\right) y_{*}(t)=0, \quad t \in \Delta,
$$

из которого немедленно следует, что функция $d(t)$ обращается в нуль на интервале $\Delta$.

3 а м е ч а н и е 1. Равенство (3.19) можно получить, непосредственно подставляя равенства

$$
L_{u}(t)=0, \quad L_{u}^{\prime}(t)=0, \quad L_{u}^{\prime \prime}(t)=0
$$

в уравнение (3.4). Тогда функция $d(t)$ также будет равна нулю, и из монотонности функции $g(z)$ находим требуемое равенство.

3 а м е ч а н и е 2. Формула (3.19) задает прямую

$$
m_{1}\left(r m_{2}-a_{21} m_{1}\right) x+m_{2}\left(r a_{12} m_{2}-m_{1}\right) y=0,
$$

которая при выполнении неравенств (3.15) или неравенств (3.16) пересекает множество

$$
\{(x, y): 0<x<1,0<y<1\} .
$$

Поэтому фазовые переменные $x_{*}(t)$ и $y_{*}(t)$ будут находиться на этой прямой, когда оптимальное управление $u_{*}(t)$ будет иметь особый режим на интервале $\Delta$.

Анализируя уравнение (3.4), мы видим, что вторая производная $L_{u}^{\prime \prime}(t)$ функции $L_{u}(t)$ не имеет слагаемых, содержащих управление $u_{*}(t)$. Значит, согласно $[18$, с. 40$]$ порядок $q$ возможного особого режима больше единицы и необходимо дальше продолжить процесс дифференцирования функции переключений $L_{u}(t)$.

Далее, вычислим третью производную $L_{u}^{\prime \prime \prime}(t)$ функции $L_{u}(t)$. Для этого будем опять использовать уравнение (3.4). Согласно равенствам (3.19) и (3.20) имеем

$$
L_{u}^{\prime \prime \prime}(t)=m_{1}^{-1} g^{\prime}\left(z_{*}(t)\right)\left[m_{1}\left(r m_{2}-a_{21} m_{1}\right) x_{*}(t)+m_{2}\left(r a_{12} m_{2}-m_{1}\right) y_{*}(t)\right]^{\prime} \phi_{2}(t), \quad t \in \Delta .
$$

Привлекая уравнения системы (1.4), выполним в формуле (3.21) требуемое дифференцирование. В результате приходим к соотношению

$$
\begin{gathered}
L_{u}^{\prime \prime \prime}(t)=m_{1}^{-1} g^{\prime}\left(z_{*}(t)\right)\left(r m_{1}\left(r m_{2}-a_{21} m_{1}\right)\left(1-x_{*}(t)-a_{12} y_{*}(t)\right) x_{*}(t)\right. \\
+m_{2}\left(r a_{12} m_{2}-m_{1}\right)\left(1-y_{*}(t)-a_{21} x_{*}(t)\right) y_{*}(t) \\
\left.-g\left(z_{*}(t)\right)\left(m_{1}^{2}\left(r m_{2}-a_{21} m_{1}\right) x_{*}(t)+m_{2}^{2}\left(r a_{12} m_{2}-m_{1}\right) y_{*}(t)\right)\right) \phi_{2}(t) .
\end{gathered}
$$


Приравняем эту производную к нулю: $L_{u}^{\prime \prime \prime}(t)=0, \quad t \in \Delta$. Благодаря лемме 2 и монотонности функции $g(z)$ из (3.22) получаем выражение

$$
\begin{gathered}
g\left(z_{*}(t)\right)\left(m_{1}^{2}\left(r m_{2}-a_{21} m_{1}\right) x_{*}(t)+m_{2}^{2}\left(r a_{12} m_{2}-m_{1}\right) y_{*}(t)\right) \\
=r m_{1}\left(r m_{2}-a_{21} m_{1}\right)\left(1-x_{*}(t)-a_{12} y_{*}(t)\right) x_{*}(t)+m_{2}\left(r a_{12} m_{2}-m_{1}\right)\left(1-y_{*}(t)-a_{21} x_{*}(t)\right) y_{*}(t) .
\end{gathered}
$$

Упростим его. Для этого подставим в (3.23) формулу

$$
y_{*}(t)=-\frac{m_{1}\left(r m_{2}-a_{21} m_{1}\right)}{m_{2}\left(r a_{12} m_{2}-m_{1}\right)} x_{*}(t),
$$

вытекающую из (3.19). После несложных преобразований находим

$$
\left(m_{2}-m_{1}\right) g\left(z_{*}(t)\right)=(1-r)+\left[\left(r-a_{21}\right)+\left(1-r a_{12}\right) \frac{m_{1}\left(r m_{2}-a_{21} m_{1}\right)}{m_{2}\left(r a_{12} m_{2}-m_{1}\right)}\right] x_{*}(t) .
$$

Поскольку существуют ограничения на фазовые переменные $x_{*}(t)$ и $z_{*}(t)$, вытекающие из леммы 1 , а также ограничения на параметры $r, a_{12}, a_{21}, m_{1}, m_{2}$ в виде неравенств (1.5), (3.15) и (3.16), то равенство (3.25) может оказаться либо противоречивым, либо нет. Тогда в первом случае исходное предположение о возможности обращения функции переключений $L_{u}(t)$ тождественно в нуль на интервале $\Delta$ становится неверным. Особый режим у оптимального управления $u_{*}(t)$ будет отсутствовать. Это управление окажется релейной функцией на всем отрезке $[0, T]$, принимающей значения 0 и $M$. Во втором случае в силу монотонности функции $g(z)$ формула (3.25) дает возможность определить фазовую переменную $z_{*}(t)$ на интервале $\Delta$.

Продолжим дифференцирование функции переключений $L_{u}(t)$ и найдем ее четвертую производную $L_{u}^{\prime \prime \prime \prime}(t)$ с помощью формулы (3.22). Согласно (3.23) в результате дифференцирования имеем

$$
\begin{gathered}
L_{u}^{\prime \prime \prime \prime}(t)=m_{1}^{-1} g^{\prime}\left(z_{*}(t)\right)\left[h\left(x_{*}(t), y_{*}(t)\right)\right. \\
\left.-g\left(z_{*}(t)\right)\left(m_{1}^{2}\left(r m_{2}-a_{21} m_{1}\right) x_{*}(t)+m_{2}^{2}\left(r a_{12} m_{2}-m_{1}\right) y_{*}(t)\right)\right]^{\prime} \phi_{2}(t), \quad t \in \Delta,
\end{gathered}
$$

где

$$
\begin{gathered}
h\left(x_{*}(t), y_{*}(t)\right)=r m_{1}\left(r m_{2}-a_{21} m_{1}\right)\left(1-x_{*}(t)-a_{12} y_{*}(t)\right) x_{*}(t) \\
+m_{2}\left(r a_{12} m_{2}-m_{1}\right)\left(1-y_{*}(t)-a_{21} x_{*}(t)\right) y_{*}(t) .
\end{gathered}
$$

Используя при дифференцировании выражения в квадратных скобках в формуле (3.26) уравнения системы (1.4) и (1.6), выводим желаемое соотношение

$$
\begin{gathered}
L_{u}^{\prime \prime \prime \prime}(t)=m_{1}^{-1} g^{\prime}\left(z_{*}(t)\right)\left(-u_{*}(t)\left(m_{1}^{2}\left(r m_{2}-a_{21} m_{1}\right) x_{*}(t)+m_{2}^{2}\left(r a_{12} m_{2}-m_{1}\right) y_{*}(t)\right)\right. \\
\left.+p\left(x_{*}(t), y_{*}(t), z_{*}(t)\right)\right) \phi_{2}(t), \quad t \in \Delta,
\end{gathered}
$$

где функция $p\left(x_{*}(t), y_{*}(t), z_{*}(t)\right)$ содержит только слагаемые, в которых управление $u_{*}(t)$ отсутствует.

Получив (3.27), проверим выполнение необходимого условия оптимальности особого режима (условие Келли - Коппа - Мойера) из [18, с. 40]. Для этого оценим множитель при управлении $u_{*}(t)$ :

$$
-\left(m_{1}^{2}\left(r m_{2}-a_{21} m_{1}\right) x_{*}(t)+m_{2}^{2}\left(r a_{12} m_{2}-m_{1}\right) y_{*}(t)\right) .
$$

Подставляя в него формулу (3.24) и затем преобразуя, находим

$$
m_{1}\left(m_{2}-m_{1}\right)\left(r m_{2}-a_{21} m_{1}\right) x_{*}(t) .
$$


Вследствие леммы 1 и неравенства (1.5) анализ этого выражения приводит к следующим выводам:

1) при $r m_{2}-a_{21} m_{1}>0$, что означает выполнение неравенств (3.15), условие Келли Коппа - Мойера не выполнено. Значит, особого режима у оптимального управления $u_{*}(t)$ не существует. Оно является релейной функцией на всем отрезке $[0, T]$, принимающей значения 0 и $M$;

2) при $r m_{2}-a_{21} m_{1}<0$, что означает выполнение неравенств (3.16), условие Келли - Коппа - Мойера выполнено, и более того, в усиленной форме (имеет место строгое неравенство). Тогда у оптимального управления $u_{*}(t)$ может существовать особый режим.

Приравнивая четвертую производную $L_{u}^{\prime \prime \prime \prime}(t)$ из (3.27) к нулю и учитывая преобразование, совершенное в (3.28), находим формулу особого режима для управления $u_{*}(t)$ :

$$
u_{\text {sing }}(t)=\frac{p\left(x_{\text {sing }}(t), y_{\text {sing }}(t), z_{\text {sing }}(t)\right)}{m_{1}\left(m_{2}-m_{1}\right)\left(r m_{2}-a_{21} m_{1}\right) x_{\text {sing }}(t)}, \quad t \in \Delta .
$$

Здесь $x_{\operatorname{sing}}(t), y_{\operatorname{sing}}(t), z_{\operatorname{sing}}(t)$ - компоненты оптимального решения $\left(x_{*}(t), y_{*}(t), z_{*}(t)\right)$ на особом режиме. Легко видеть, что такая формула задает управление $u_{\operatorname{sing}}(t)$ в виде обратной связи, т. е. в виде зависимости только от $x_{\text {sing }}(t), y_{\text {sing }}(t), z_{\text {sing }}(t)$. Естественно предполагать, что это управление является допустимым всюду на интервале $\Delta$. Также понятно, что если управление $u_{\operatorname{sing}}(t)$ оказывается недопустимым на этом интервале, то снова приходим к выводу об отсутствии особого режима у оптимального управления $u_{*}(t)$. Тогда управление $u_{*}(t)$ опять является релейной функцией на отрезке $[0, T]$, принимающей значения 0 и $M$.

Обсудим теперь расположение интервала $\Delta$ на отрезке $[0, T]$. Важен вопрос о том, может ли этот интервал примыкать к концу $T$ отрезка $[0, T]$. Справедлива следующая лемма.

Лемма 4. Пусть имеет место неравенство

$$
\alpha \neq-\frac{r m_{2}-a_{21} m_{1}}{r a_{12} m_{2}-m_{1}} .
$$

Тогда интервал $\Delta$, на котором оптимальное управление $u_{*}(t)$ может иметь особый режим, не примыкает к кониу $T$ отрезка $[0, T]$.

Д ок а з а т е ль с т в о. Предположим противное. Пусть интервал $\Delta$ примыкает к $T$. Тогда из формулы (3.24) при $t=T$ получаем

$$
\frac{m_{2}}{m_{1}} \cdot \frac{y_{*}(T)}{x_{*}(T)}=-\frac{r m_{2}-a_{21} m_{1}}{r a_{12} m_{2}-m_{1}} .
$$

Из уравнения (3.1), взятого при $t=T$, и начального условия (3.2) вытекает соотношение

$$
L_{u}^{\prime}(T)=-m_{1}^{-1} g^{\prime}\left(z_{*}(T)\right) x_{*}(T)\left[-\alpha+\frac{m_{2}}{m_{1}} \cdot \frac{y_{*}(T)}{x_{*}(T)}\right] .
$$

Подставляя в него (3.30), имеем

$$
L_{u}^{\prime}(T)=-m_{1}^{-1} g^{\prime}\left(z_{*}(T)\right) x_{*}(T)\left[-\alpha-\frac{r m_{2}-a_{21} m_{1}}{r a_{12} m_{2}-m_{1}}\right] .
$$

Благодаря монотонности функции $g(z)$, лемме 1 и (3.29) мы приходим к противоречивому заключению о том, что $L_{u}^{\prime}(T) \neq 0$. Значит наше предположение неверно. Утверждение доказано.

Будем предполагать в дальнейших рассуждениях, что неравенство (3.29) выполнено. Тогда, как следует из леммы 4 , к концу $T$ отрезка $[0, T]$ примыкает интервал, на котором функция переключений $L_{u}(t)$ принимает либо только положительные, либо только отрицательные значения. Следовательно, далее важно обсудить вопрос о том, каким образом происходит соединение интервала $\Delta$ (особого участка), на котором оптимальное управление $u_{*}(t)$ может иметь 
особый режим, с неособыми участками, на которых это управление релейно. Именно считая, что на интервале $\Delta$ управление $u_{\operatorname{sing}}(t)$ принимает значения только из интервала $(0, M)$ и привлекая теорему А.3.3 [17], мы заключаем, что соединение между интервалом $\Delta$ с заданным на нем управлением $u_{\operatorname{sing}}(t)$ и неособыми участками, где оптимальное управление $u_{*}(t)$ является релейной функцией и принимает значения 0 и $M$, возможно и данные соединения могут происходить с обеих концов интервала $\Delta$. Кроме того, такие неособые участки содержат счетное число переключений управления $u_{*}(t)$, которые накапливаются к соответствующим точкам соединения особого и неособого участков. У этих точек соединения возникает такое явление, как четтеринг [16, гл. $2.11 ; 17$, с. $401 ; 18]$.

3 а м е ч а н и е 3 . Понятно, что оптимальное управление $u_{*}(t)$, содержащее четтеринг, не может являться эффективной стратегией лечения рассматриваемых заболеваний. Поэтому в случае его возникновения могут быть использованы некоторые способы аппроксимации с помощью кусочно-постоянных управлений, представленных в [17, гл. 6.3] и [20-23].

Таким образом, на основании проделанных рассуждений мы приходим к следующему выводу.

Утверждение. Оптимальное управление $u_{*}(t)$ может быть одного из следующих четырех видов:

- постоянной функцией, принимающей значение 0 или $M$;

- кусочно-постоянной функцией с одним переключением типа (3.13) или (3.14);

- релейной функиией с конечным числом переключений, принимаюоей значения 0 и $\mathrm{M}$;

- функиией, содержащей особый режим на особом участке, который при помощи четтеринга соединяется с неособыми, релейными участками.

\section{4. Результаты численных расчетов}

Теперь мы продемонстрируем результаты численных расчетов, выполненных с использованием среды ВОСОР-2.0.5 [24]. Она представляет собой специальную среду, реализованную в MATLAB, для решения задач оптимального управления с общими концевыми и фазовыми ограничениями со свободным или фиксированным конечным временем. После дискретизации по времени такие задачи аппроксимируются конечномерными задачами оптимизации, которые затем решаются с помощью хорошо известного программного обеспечения IPOPT, использующего точно вычисленные производные благодаря ADOL-C. При этом IPOPT является программным пакетом с открытым исходным кодом для решения задач нелинейной оптимизации большой размерности.

Рассматривая временной интервал в 30 и 50 дней, мы использовали временную сетку с 5000 узлами. Поскольку наша задача решалась прямым методом, а значит итерационно, мы требовали на каждом шаге точность $\varepsilon=10^{-14}$. Кроме того, мы применяли правило дискретизации Лобатто III C шестого порядка. Такие особенности более подробно описаны в [24].

На графиках ниже (см. рис. 1 и 2) показаны результаты для двух случаев наборов параметров. Для каждого случая приведены оптимальное управление $u_{*}(t)$, отвечающие ему оптимальные траектории $x_{*}(t)$ и $y_{*}(t)$ и концентрация химиотерапевтического препарата $z_{*}(t)$. Заметим, что в представленных расчетах она также является функцией терапии $(g(z)=z)$. Ниже располагаются фазовые портреты для каждого случая.

Черные сплошные линии отражают оптимальные решения на выбранном отрезке времени лечения $[0, T]$. Для иллюстрации преимуществ лечения серыми пунктирными линиями приведено решение неуправляемой системы (1.2), т. е. траектории при отсутствии лечения $(u(t) \equiv 0)$.

На рис. 1 представлен случай, когда выражения $\left(r m_{2}-a_{21} m_{1}\right)$ и $\left(r a_{12} m_{2}-m_{1}\right)$ удовлетворяют неравенствам (3.7). В этом случае, как следует из предшествующего анализа, оптимальное управление $u_{*}(t)$ имеет вид (3.13). Сравним оптимальные траектории и траектории, полученные в результате отсутствия лечения. Нетрудно видеть, что оптимальное управление $u_{*}(t)$ 

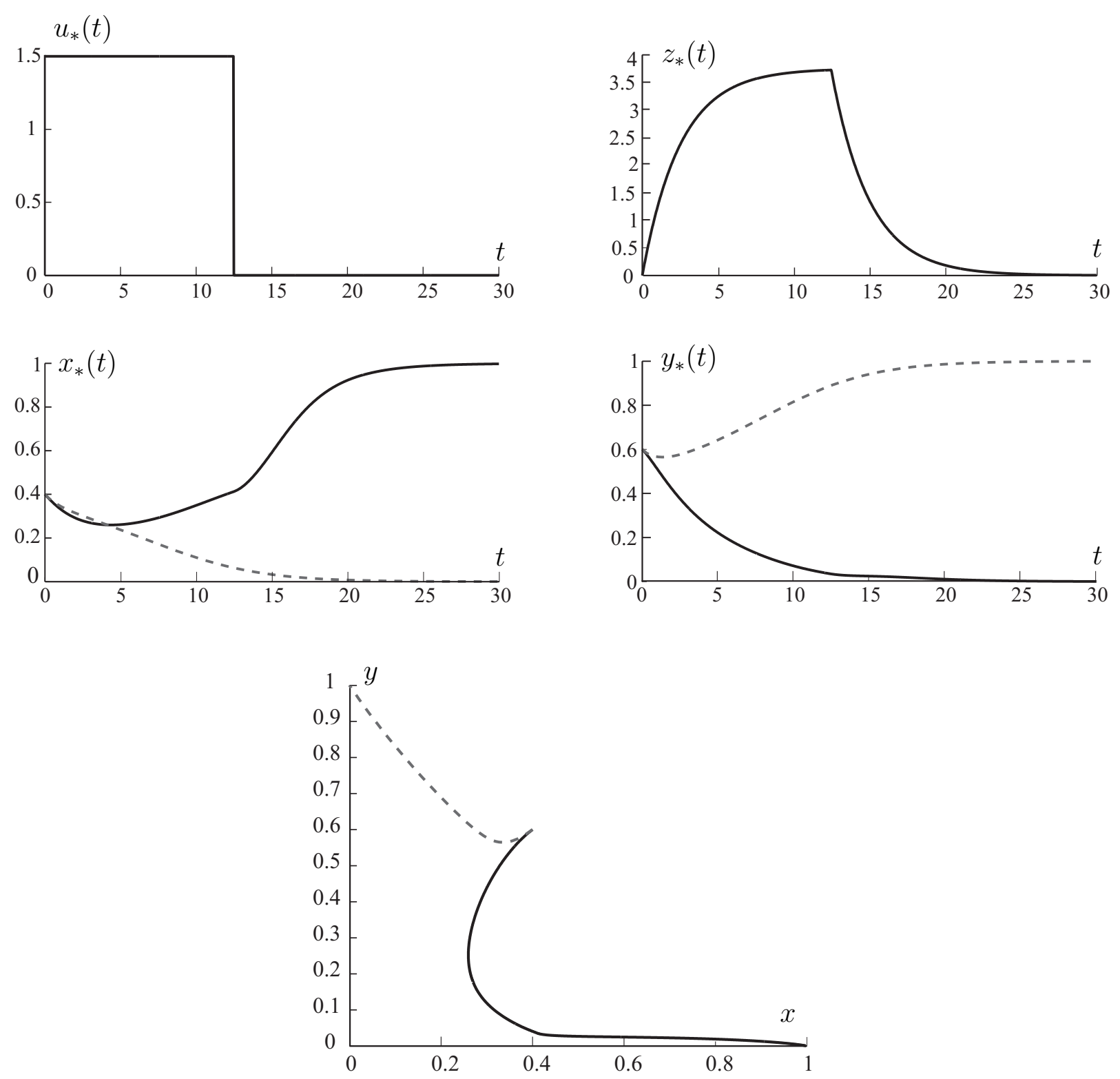

Рис. 1. Первый случай набора параметров. Начальные условия $x(0)=0.4, y(0)=0.6$. Коэффициенты $r=0.8, a_{12}=1.4, a_{21}=1.3, m_{1}=0.1, m_{2}=0.2, \gamma=0.4, \alpha=1.2, M=1.5, T=30$. Значение функционала $J_{*}=-1.19664$

переводит траекторию системы из области притяжения устойчивого положения равновесия на вертикальной оси (что соответствует вымиранию здоровых клеток) в область притяжения устойчивого положения равновесия на горизонтальной оси (что соответствует вымиранию раковых клеток). В данном случае лечение приводит к полному выздоровлению пациента, "перетягивая" траекторию в выгодное для этого устойчивое положение равновесия.

На рис. 2 рассмотрим случай, когда выражения $\left(r m_{2}-a_{21} m_{1}\right)$ и $\left(r a_{12} m_{2}-m_{1}\right)$ удовлетворяют неравенствам (3.16). В представленных расчетах оптимальное управление $u_{*}(t)$ имеет особый режим на особом участке, который при помощи четтеринга соединяется с неособыми, релейными участками, что полностью согласуется с полученным теоретическим результатом. Здесь так же, как и в предыдущем примере, оптимальное управление $u_{*}(t)$ переводит траекторию системы из области притяжения устойчивого положения равновесия на вертикальной оси в область притяжения устойчивого положения равновесия на горизонтальной оси. Это означает, что лечение приводит к полному выздоровлению пациента, “перетягивая" траекторию в 

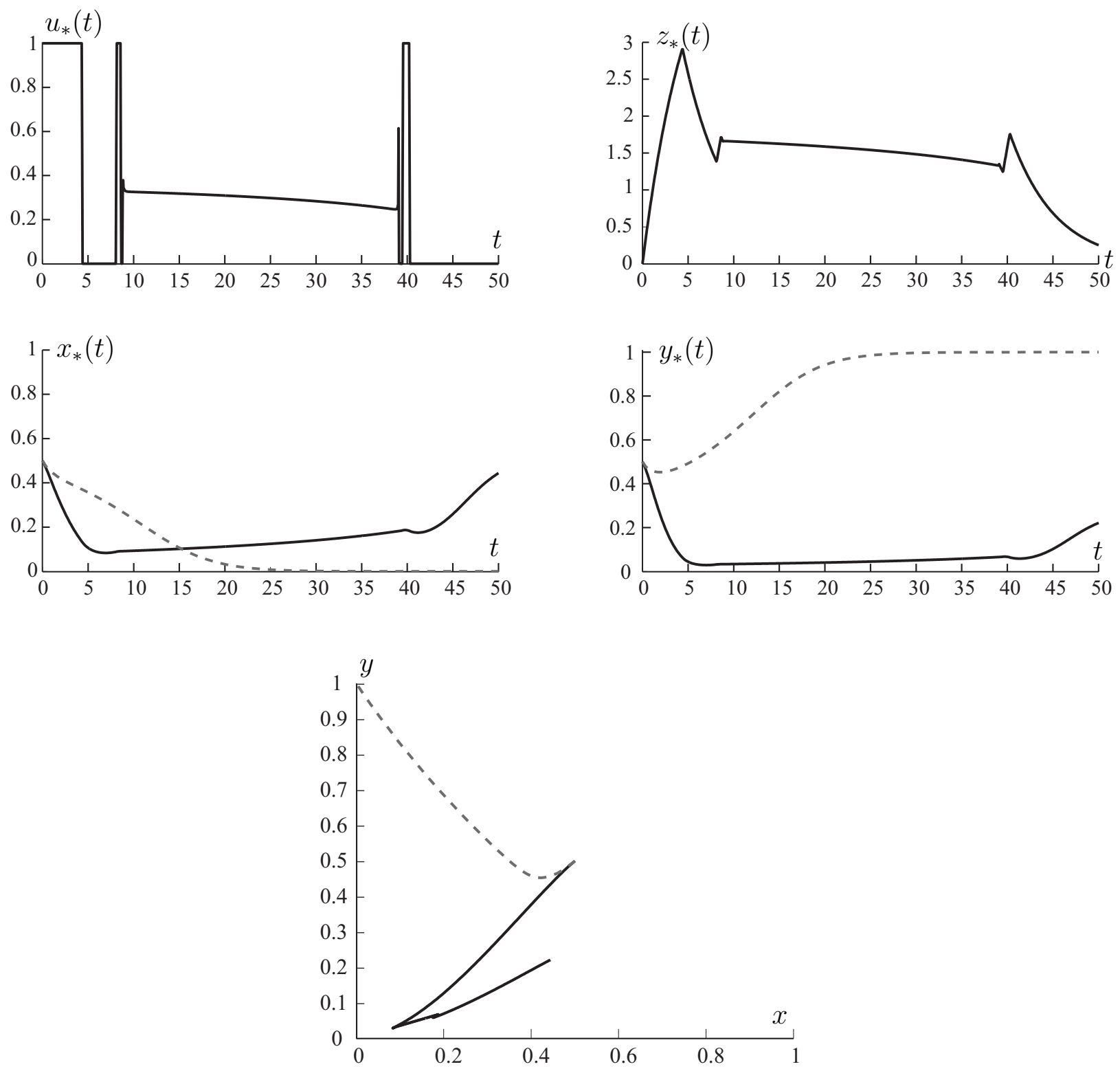

Рис. 2. Второй случай набора параметров. Начальные условия $x(0)=0.5, y(0)=0.5$. Коэффициенты $r=0.6, a_{12}=1.5, a_{21}=1.3, m_{1}=0.3, m_{2}=0.5, \gamma=0.2, \alpha=1.0, M=1.0, T=50$. Значение функционала $J_{*}=-0.222657$

выгодное для этого устойчивое положение равновесия. Такой вывод подтверждают расчеты для $T=100$.

\section{СПИСОК ЛИТЕРАТУРЫ}

1. Todorov Y., Fimmel E., Bratus A.S., Semenov Y.S., Nuernberg F. A optimal strategies for leukemia therapy: a multi-objective approach // Russ. J. Numer. Anal. Math. Model. 2011. Vol. 26, no. 6. P. 589-604. doi: 10.1515/rjnamm.2011.035.

2. Bratus A.S., Fimmel E., Todorov Y., Semenov Y.S., Nürnberg F. On strategies on a mathematical model for leukemia therapy // Nonlinear Analysis: Real World Appl. 2012. Vol. 13, no. 3. P. 1044-1059. doi: 10.1016/j.nonrwa.2011.02.027.

3. Bratus A.S., Goncharov A.S., Todorov I.T. Optimal control in a mathematical model for leukemia therapy with phase constraints // Moscow Univ. Comput. Math. Cybern. 2012. Vol. 36, no. 4. P. 178-182. doi: $10.3103 / \mathrm{S} 0278641912040024$. 
4. Bratus A., Todorov Y., Yegorov I., Yurchenko D. Solution of the feedback control problem in the mathematical model of leukemia therapy // J. Optim. Theory Appl. 2013. Vol. 159, no. 3. P. 590-605. doi: 10.1007/s10957-013-0324-6.

5. Fimmel E., Semenov Y.S., Bratus A.S. On optimal and suboptimal treatment strategies for a mathematical model of leukemia // Math. Biosci. Eng. 2013. Vol. 10, no. 1. P. 151-165. doi: $10.3934 /$ mbe.2013.10.151 .

6. Egorov I.E. Assessing alternative control strategies for systems with asymptotically stable equilibrium positions // Moscow Univ. Comput. Math. Cybern. 2013. Vol. 37, no. 3, P. 112-120. doi: 10.3103/S0278641913030059.

7. Solé R.V., Deisboeck T.S. An error catastrophe in cancer? // J. Theor. Biol. 2004. Vol. 228. P. $47-54$. doi 10.1016/j.jtbi.2003.08.018.

8. Solé R.V., Garcia I.G., Costa J. Spatial dynamics in cancer // Complex Systems Science in Biomedicine / eds. T.S. Deisboeck, J.Y. Kresh. N Y: Springer, 2006. P. 557-572. (Topics in Biomedical Engineering International Book Series.) doi 10.1016/j.jtbi.2003.08.018.

9. Кучумов А.Г. Математическое моделирование и биомеханический подход к описанию развития, диагностике и лечения онкологических заболеваний // Российский журнал биомеханики. 2010. T. 14, № 4. C. 42-69.

10. Khailov E.N., Klimenkova A.D., Korobeinikov A. Optimal control for anticancer therapy // Extended abstracts spring 2018 / eds. A. Korobeinikov, M. Caubergh, T. Lázaro, J. Sardanyés. Basel: Birkhäuser, 2019. P. 35-43. (Trends in mathematics; vol. 11). doi: 10.1007/978-3-030-25261-8_6.

11. Братусь А.С., Новожилов А.С., Платонов А.П. Динамические системы и модели биологии. М.: Физматлит, 2010. 400 с.

12. Тарасевич Ю.Ю. Математическое и компьютерное моделирование. Вводный курс. М.: Либроком, 2013. 152 c.

13. Хартман Ф. Обыкновенные дифференциальные уравнения. М.: Мир, 1970. 720 с.

14. Ли Э.Б., Маркус Л. Основы теории оптимального управления. М.: Наука, 1972. 576 с.

15. Васильев Ф.П. Методы оптимизации. М.: Факториал Пресс, 2002. 824 с.

16. Schättler H., Ledzewicz U. Geometric optimal control: theory, methods and examples. N Y; Heidelberg; Dordrecht; London: Springer, 2012. 640 p.

17. Schättler H., Ledzewicz U. Optimal control for mathematical models of cancer therapies: an applications of geometric methods. N Y; Heidelberg; Dordrecht; London: Springer, 2015. 496 p. doi: 10.1007/978-1-4939-2972-6.

18. Zelikin M.I., Borisov V.F. Theory of chattering control with applications to astronautics, robotics, economics, and engineering. Boston: Birkhäuser, 1994. 244 p. doi: 10.1007/978-1-4612-2702-1.

19. Левин А.Ю. Неосцилляция решений уравнения $x^{n}+p_{1}(t) x^{n-1}+\cdots+p_{n}(t) x=0 / /$ Успехи мат. наук. 1969. Т. 24, вып. 2. С. 43-96.

20. Зеликин М.И., Зеликина Л.Ф. Уклонение функционала от оптимального значения при четтеринге экспоненциально убывает с ростом числа переключений // Дифференц. уравнения. 1999. T. 35, № 11. C. 1468-1472.

21. Zhu J., Trélat E., Cerf M. Planar titling maneuver of a spacecraft: singular arcs in the minimum time problem and chattering // Discrete Cont. Dyn. Ser. B. 2016. Vol. 21, no. 4. P. 1347-1388. doi: 10.3934/dcdsb.2016.21.1347.

22. Yegorov I., Mairet F., Gouzé J.-L. Optimal feedback strategies for bacterial growth with degradation, recycling, and effect of temperature // Optim. Control Appl. Meth. 2018. Vol. 39, no. 2. P. 1084-1109. doi: 10.1002/oca.2398.

23. Grigorieva E., Khailov E. Chattering and its approximation in control of psoriasis treatment // Discrete Cont. Dyn. Ser. B. 2019. Vol. 24, no. 5. P. 2251-2280. doi: 10.3934/dcdsb.2019094.

24. Bonnans F., Martinon P., Giorgi D., Grélard V., Maindrault S., Tissot O., Liu J. BOCOP 2.0.5 - User guide [e-resource]. 2017. URL: http://bocop.org .

Поступила 16.01.2020

После доработки 28.01.2020

Принята к публикации 3.02.2020

Григоренко Николай Леонтьевич

д-р физ.-мат. наук, профессор

фак. ВМК МГУ им. М.В. Ломоносова 


\author{
г. Москва \\ e-mail: grigor@cs.msu.su
}

Хайлов Евгений Николаевич

канд. физ.-мат. наук, доцент

фак. ВМК МГУ им. М.В. Ломоносова

г. Москва

e-mail: khailov@cs.msu.su

Григорьева Эллина Валерьевна

канд. физ.-мат. наук, профессор

Техасский женский университет, США

e-mail: egrigorieva@mail.twu.edu

Клименкова Анна Дмитриевна

студент

фак. ВМК МГУ им. М.В. Ломоносова

г. Москва

e-mail: klimenkovaad@mail.ru

\title{
REFERENCES
}

1. Todorov Y., Fimmel E., Bratus A., Semenov Y., Nuernberg F. An optimal strategy for leukemia therapy: a multi-objective approach. Russ. J. Numer. Anal. Math. Model., 2011, vol. 26, no. 6, pp. 589-604. doi: $10.1515 /$ rjnamm.2011.035.

2. Bratus A.S., Fimmel E., Todorov Y., Semenov Y.S., Nürnberg F. On strategies on a mathematical model for leukemia therapy. Nonlinear Analysis: Real World Appl., 2012, vol. 13, no. 3, pp. 1044-1059. doi: 10.1016/j.nonrwa.2011.02.027.

3. Bratus A.S., Goncharov A.S., Todorov I.T. Optimal control in a mathematical model for leukemia therapy with phase constraints. Moscow Univ. Comput. Math. Cybern., 2012, vol. 36, no. 4, pp. 178182. doi: $10.3103 / \mathrm{S} 0278641912040024$.

4. Bratus A., Todorov Y., Yegorov I., Yurchenko D. Solution of the feedback control problem in the mathematical model of leukaemia therapy. J. Optim. Theory Appl., 2013, vol. 159, no. 3, pp. 590-605. doi: 10.1007/s10957-013-0324-6 .

5. Fimmel E., Semenov Y., Bratus A. On optimal and suboptimal treatment strategies for a mathematical model of leukemia. Math. Biosci. Eng., 2013, vol. 10, no. 1, pp. 151-165. doi: 10.3934/mbe.2013.10.151.

6. Egorov I.E. Assessing alternative control strategies for systems with asymptotically stable equilibrium positions. Moscow Univ. Comput. Math. Cybern., 2013, vol. 37, no. 3, pp. 112-120. doi: $10.3103 / \mathrm{S} 0278641913030059$.

7. Solé R.V., Deisboeck T.S. An error catastrophe in cancer? J. Theor. Biol., 2004, vol. 228, pp. 47-54. doi 10.1016/j.jtbi.2003.08.018.

8. Solé R.V., Garcia I.G., Costa J. Spatial dynamics in cancer. In: Deisboeck T.S., Kresh J.Y. (eds), Complex Systems Science in Biomedicine. Topics in Biomedical Engineering International Book Series. N Y: Springer, 2006, pp. 557-572. doi: 10.1007/978-0-387-33532-2_24.

9. Kuchumov A.G. Mathematical modelling and biomechanical approach to describe the development, the diagnostics, and the treatment of oncological diseases. Russian Journal of Biomechanics, 2010, vol. 14, no. 4, pp. 42-69. (in Russian)

10. Khailov E.N., Klimenkova A.D., Korobeinikov A. Optimal control for anticancer therapy. In: Korobeinikov A., Caubergh M., Lázaro T., Sardanyés J. (eds), Extended Abstracts Spring 2018. Ser. Trends in Mathematics, vol. 11. Basel: Birkhäuser, 2019, pp. 35-43. doi: 10.1007/978-3-030-25261-8_6 .

11. Bratus' A.S., Novozhilov A.S., Platonov A.P. Dinamicheskie sistemy i modeli biologii [Dynamic systems and models in biology]. Moscow: Fizmatlit Publ., 2010, 400 p. ISBN: 978-5-9221-1192-8.

12. Tarasevich Yu.Yu. Matematicheskoe i komp'yuternoe modelirovanie: Vvodnyi kurs [Mathematical and computer modeling: Introductory course]. Moscow: Librokom Publ., 2013, 152 p.

ISBN: 978-5-397-03828-7.

13. Hartman Ph. Ordinary differential equations. N Y: John Wiley \& Sons, 1964, 612 p. Translated to Russian under the title Obyknovennye differentsial'nye uravneniya. Moscow: Mir Publ., 1970, 720 p. 
14. Lee E.B., Markus L. Foundations of optimal control theory. N.Y.; London; Sydney: John Wiley \& Sons, Inc., 1967, 576 p. ISBN: 9780471522638 . Translated to Russian under the title Osnovy teorii optimal'nogo upravleniya, Moscow: Nauka Publ., 1972, 576 p.

15. Vasil'ev F.P. Metody optimizatsii [Optimization methods]. Moscow: Factorial Press, 2002, 824 p. ISBN: 5-88688-056-9 .

16. Schättler H., Ledzewicz U. Geometric optimal control: theory, methods and examples. N Y; Heidelberg; Dordrecht; London: Springer, 2012, 640 p. ISBN: 978-1-4614-3834-2 .

17. Schättler H., Ledzewicz U. Optimal control for mathematical models of cancer therapies: an applications of geometric methods. N Y; Heidelberg; Dordrecht; London: Springer, 2015, 496 p. doi: 10.1007/978-14939-2972-6.

18. Zelikin M.I., Borisov V.F. Theory of chattering control with applications to astronautics, robotics, economics, and engineering. Boston: Birkhäuser, 1994, 244 p. doi: 10.1007/978-1-4612-2702-1.

19. Levin A.Yu. Non-oscillation of solutions of the equation $x^{n}+p_{1}(t) x^{n-1}+\cdots+p_{n}(t) x=0$. Russian Math. Surveys, 1969, vol. 24, no. 2, pp. 43-99. doi: 10.1070/RM1969v024n02ABEH001342.

20. Zelikin M.I., Zelikina L.F. The deviation of a functional from its optimal value under chattering decreases exponentially as the number of switchings grows. Differ. Equ., 1999, vol. 35, no. 11, pp. 1489-1493.

21. Zhu J., Trélat E., Cerf M. Planar titling maneuver of a spacecraft: singular arcs in the minimum time problem and chattering. Discrete Cont. Dyn.-B, 2016, vol. 21, no. 4, pp. 1347-1388. doi: $10.3934 /$ dcdsb.2016.21.1347.

22. Yegorov I., Mairet F., Gouzé J.-L. Optimal feedback strategies for bacterial growth with degradation, recycling, and effect of temperature. Optim. Control Appl., 2018, vol. 39, no. 2, pp. 1084-1109. doi: 10.1002/oca.2398 .

23. Grigorieva E., Khailov E. Chattering and its approximation in control of psoriasis treatment. Discrete Contin. Dyn. Syst., Ser. B, 2019, vol. 24, no. 5, pp. 2251-2280. doi: 10.3934/dcdsb.2019094.

24. Bonnans F., Martinon P., Giorgi D., Grélard V., Maindrault S., Tissot O., Liu J. BOCOP 2.0.5 - user guide. 2017. Available at: URL: http://bocop.org .

Received January 16, 2020

Revised January 28, 2020

Accepted February 3, 2020

Funding Agency: The work of the first two authors was supported by the Russian Foundation for Basic Research jointly with the Department of Science and Technology of the Government of India (project no. 18-51-45003 IND_a).

Nikolai Leont'evich Grigorenko, Dr. Phys.-Math. Sci., Prof., Faculty of Computational Mathematics and Cybernetics, Moscow State Lomonosov University, Moscow, 119992, Russia, e-mail: grigor@cs.msu.su .

Evgenii Nikolaevich Khailov, Cand. Sci. (Phys.-Math.), Faculty of Computational Mathematics and Cybernetics, Moscow State Lomonosov University, Moscow, 119992, Russia,

e-mail: khailov@cs.msu.su .

Ellina Valer'evna Grigorieva, Cand. Sci. (Phys.-Math.), Prof., Department of Mathematics and Computer Sciences, Texas Woman's University, Denton, TX 76204, USA,

e-mail: egrigorieva@mail.twu.edu .

Anna Dmitrievna Klimenkova, undergraduate student, Faculty of Computational Mathematics and Cybernetics, Moscow State Lomonosov University, Moscow, 119992, Russia, e-mail: klimenkovaad@mail.ru .

Cite this article as: N.L. Grigorenko, E. N. Khailov, E. V. Grigorieva, A. D. Klimenkova. Optimal strategies in the treatment of cancers in the Lotka-Volterra mathematical model of competition. Trudy Instituta Matematiki i Mekhaniki URO RAN, 2020, vol. 26, no. 1, pp. 71-88. 